\title{
Study on the Development Direction of Power Grid Dispatching Technical Support System
}

\author{
Renbo Wu' ${ }^{1}$, Hong Luo ${ }^{2}$, Rui $\mathrm{Qi}^{1}$, Ming Gao ${ }^{1}$, Chao Liang ${ }^{2}$ \\ 1 Guangzhou Power Supply Co.,Ltd., Guangzhou 510000,China \\ 2 Yunnan Yundian Tongfang Technology Co.,Ltd., Kunming 650000,China
}

\begin{abstract}
In order to ensure the safe, stable operation of Guangzhou power grid to meet the large city power grid requirements, reached the international advanced level, to realize the transformation of Guangzhou electric power dispatching control center strategy and control mode, it requires the construction of a new generation of intelligent dispatching technical support system. This paper analyzes the current situation and development of Guangzhou power grid. Based on the rapid development of smart grid technology and information technology, this paper puts forward the development direction of power grid dispatching technology support system. It also puts forward the construction work of reasonable planning, makes clear about construction content and technical support system to meet the overall business needs "integration of regulation and control, the integration of main distribution network, market-oriented support". And we make theoretical exploration and practice and accumulated experience for the new generation of power dispatching intelligent technical support system development.
\end{abstract}

\section{Introduction}

With the rapid development of urbanization in developing countries, large urban power grid is emerging. Take Guangzhou in China for example, with the rapid development of the social economy in Guangzhou City, the scale of Guangzhou grid has been expanding, the operating characteristics have been increasingly complex, load record has got new high points, daily load curve has changed quickly, they fully embodies the characteristics of large urban power grid. In 2011, the city of Guangzhou as a large grid is peeled off from the provincial administrative regions and got separate operations, the demand for electricity will continue to grow and it has a higher reliability rate for power supply requirements to ensure the main network security and it put forward higher requirements for power grid management.

Guangzhou is one of China's economic developed cities, its automation and intelligent development level is also in the forefront of all cities in China. With the indepth construction of Guangzhou power grid smart grid, it is bound to put forward higher automation and intelligence requirements for supporting grid dispatch technical support system [1].

In the combination of power automation and information technology, with the development and gradually mature of big data and cloud computing, it also provides technical support for the smart grid under intelligent scheduling and technical support system construction of large urban power grids.

\section{Power Grids Situation of Large City}

Guangzhou grid is an important part of the China Southern Power Grid, it is a typical power supply by the end of the grid, set the "provincial, prefectural and county (with)" multi-level scheduling as a whole, it is responsible for direct dispatch control center within the scope of Guangzhou grid jurisdiction, substation and power plants, transfer tube covering power system ", transmission, transformation, distribution, use the" various production processes, there are nearly 300 substations, nearly 20 power plants, covering $500 \mathrm{kV}$ voltage level to $10 \mathrm{kV}$, which includes water energy, fire and new energy.

Currently, the most power dispatch technical support system is connected to the backbone network, including the main network and distribution network technical support systems, they are used to support the operation monitoring grid and distribution network, control, management and other services. They play a significant role in network operation and management control. These systems are relative isolation among each other, data sharing is difficult. Due to the limitations of grid size and technical positioning system construction time, there are problems in the system of real-time static and dynamic analysis, decision support, intelligent alarms, maintenance optimization and planning smart layout. FIG modulus management and other aspects lack of capacity, there are significant gaps compared with developed countries. Take Guangzhou power grid for example, 
because of their function transformation and upgrading, automation systems and their operational management and decision support systems cannot meet the power requirements of operational control and management and the degree of intelligence is very low.

\section{The Status of External Power Dispatch}

In the power system operation, the scheduling at all levels needs business exchanges of grid companies, grid scheduling mechanism in large cities also need to deal with the provincial, prefectural and county (with) multilevel scheduling service, which makes scheduling mechanism requires different business systems interact with the data grid company business collaboration. Take Guangzhou power grid for example, in addition to external electrical energy management system (EMS) and grid operation management system, Guangzhou business management also keep in touch with Guangzhou Power Supply Bureau enterprise systems (such as production management information systems, marketing, management information systems, etc.) or use the interactive functions of the system. Since the management system is not perfect, the system construction is not standardized and the limitations of the information model differences and other factors, there is a big difficulty in these data exchange and business synergies across business units [2].

As an important development strategy under the new environment of China's extra-large grid operation, the smart grid needs to be improved in routine maintenance and fault diagnosis capabilities of its dispatching technical support system. In accordance with the working ideas of the State Grid Corporation of "promoting the comprehensive construction of the three major systems and accelerating the development of a strong smart grid", improving the diagnostic and maintenance capabilities of the technical support system is a basic requirement for intelligent dispatching. At present, there are two main methods for monitoring the running status of the technical support system and the operating state of the power grid: using the alarm module provided by the technical support system base platform; and passing the general alarm monitoring software. The alarm module provided by the technical support system monitors the running status of the power grid through the collection of alarm information. After an alarm event occurs, the fault diagnosis and processing mainly depends on the operation experience of the dispatching and operation and maintenance personnel. There is a lack of technical means for pre-judging the technical support system or grid fault diagnosis and related processing plans. The fault handling often takes a long time and affects. Dispatching production; general alarm monitoring software mainly judges the running status of the grid and technical support system by monitoring the running status of individual objects. The judgment is based on a single, and it is necessary to manually analyze the alarm information of each monitoring object to infer the operation of the grid and technical support system. Status, there are faults and false negatives.

\section{Situation of Technical Support System}

Due to large cities tend to set the grid "provincial, prefectural and county (with)" multi-level scheduling functions as a whole, it needs to complete the multi-level coordination of grid operation and its management will change with the development of grid-scale. Take Guangzhou grid for example, the power grid company reform, "Group operation, integrated management," as well as other business development strategic all required technical support systems to strengthen the integration of grid control support and decision support for power grid operation, the dispatching operation form includes:

In 2012, the Southern network put forward specific demands for the regulation of the integration work, after scheduling paradigm shift, with the new regulatory requirements such as provincial regulation integration, secondary device integration, it requires power grid dispatch technical support system is able to adapt to innovation management model, support the integration of the provincial, prefectural and county (with) for network operation and management control of the business.

In large cities, the development of grid dispatch technical support system should meet the primary needs with integrated business features. Currently, the majority of domestic and foreign large-scale power is outage, one of the main reasons is the main distribution network information asymmetry and the mutual sharing of information is not timely. Through the establishment of the main distribution network information sharing and business collaboration channels, it can improve the ability to quickly avoid risks.

In large cities the grid, dispatching work should pay attention to both large grid security and stability issues, but also concerned about $110 \mathrm{kV}$ and below power grid reliability and power quality problems; we must also consider the new energy access problems caused by penetration on the safe operation of power. And there is an inherent flaw which is difficult to be resolved in the support capacity of the existing support system. The feature of "a move would be a problem" still exists and the improvement of the management level of standardization does not completely eliminate the risk. With the aging systems, new energy risk of continued access, power system operation is gradually increased.

2013, the State Council clearly stated, "promote the large consumers direct purchase and sale of electricityside power system reform." Scheduling mechanism is the executive of electricity trading result who established the electricity market and it is bound to have a profound impact on power dispatching work in many areas of power generation and real-time scheduling and other regulation. It needs full consideration of safety, energy, economic, environmental and other factors. With the advance of the electricity market, electricity trading period will be shorter and shorter, which put forward higher requirements for dispatch technical support system in data processing, decision support and functional adaptation aspects [3].

In the secondary power system construction, it will gradually break existing technical support system architecture of Guangzhou power network to establish a 
systematic and professional, service-oriented secondary power integrated system. In the support of serviceoriented technology architecture (SOA), power dispatching agencies between internal scheduling mechanism, as well as data sharing and business collaboration dispatch agencies and other business units will become faster and more efficient.

Smart grid development is the common choice of all countries of the power industry. As a large grid city, with the development and application of intelligent sensors collection terminals, heterogeneous data integration, mass data storage, high-speed computing and communications, multi-dimensional visualization technology in power grid construction and power grid intelligent level will gradually increase. With corresponding integrated analysis, wide-area control, intelligent decision support applications such as in-depth research and it will be applied to the production process and it has made considerable construction results. In-depth process of building a smart grid, the grid large cities like Guangzhou need future research at the same time with the support of intelligent scheduling management and technology systems and establish a "automation and information integration, intelligent technology to give widely used in "intelligent system to support the regulation of safety management and integration of new energy access.

With the development of smart grid, through intelligent sensors and communications network based electricity construction of things, it forms a mass of data grid operation. By big data analytics and cloud computing, it is applied to the grid operation and scheduling management, in-depth analysis and mining grid operation data, providing technical platform support for the intelligent fault diagnosis of electrical equipment, maintenance scheduling intelligent arrangement; it also be applied to the power system accident inversion and operating trends presentation and so on [4].

\section{Technical Support System Planning}

Set enterprise development as the overall grid requirements, business strategy driven by power grid dispatch technical support system for the planning and construction of power grid companies and power dispatch development; through power grid dispatch technical support system, curing business processes, improve management efficiency. On one hand, grid enterprise development strategies, specifically requested technical support for system construction and development, under the general framework of enterprise information construction of power grid, power grid dispatch technical support system construction work plan reasonable, clear grid dispatch technical support system construction content and positioning, a clear technical support system construction of key technologies; on the other hand, research the developments in smart grid technology such as big data analytics and cloud computing technology, communications technology and other technologies, these technologies raise new technologies in power network dispatching applications program, and to demonstrate.
Take Guangzhou power grid for example, make a preliminary planning for dispatch technical support system construction and development of large urban grid, including the main distribution network integration technology support system construction, supporting standard systems construction and operation and maintenance management and control system construction of the three areas its development blueprint as Fig. 1 below:
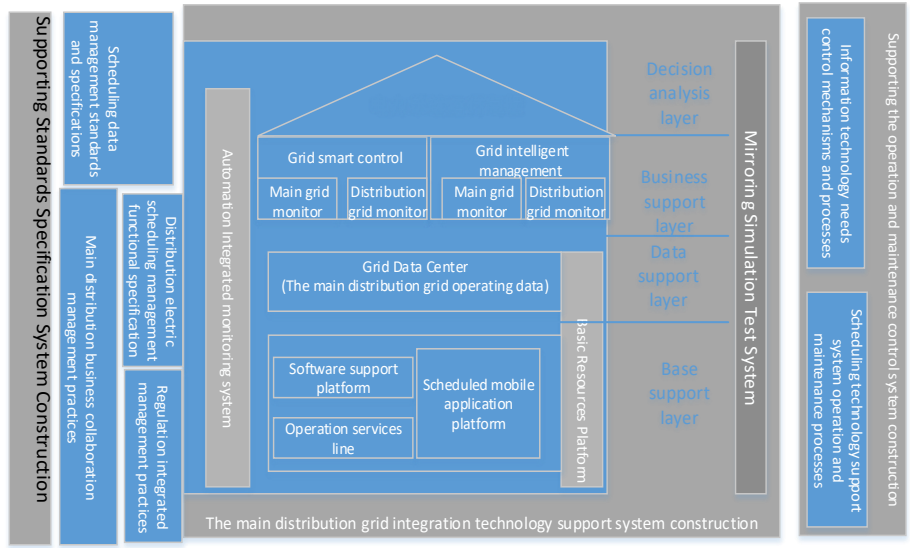

Figure 1. Development Blueprint

The main distribution network integration technical support systems mainly research the development of smart grid construction to adapt to meet the large city power grid operation control and dispatch technical support intelligent management system. The core comprising: a cockpit city power grid, power grid intelligent control system, intelligent power system operation management system, integrated foundation support platform, power grid data automation and comprehensive supervision system;

Standards system mainly includes scheduling data resource management standards and norms, the main distribution network management practices and other aspects of business collaboration and standardize business data and business processes and the application process data;

Operation and maintenance control system mainly includes the grid management information construction and management and control mechanisms and processes, scheduling technology support system operation and maintenance and other aspects of the construction process, optimize and enhance power dispatch technical support system operation and maintenance control system.

Building institutional set its schedule to meet the security and stability calculations, protection setting calculation, short circuit calculation and other advanced analysis software, maintenance management and to meet the schedule log management, fixed equipment account and a single parameter value management and other management services. In order to achieve the maintenance of equipment and parameters of the ledger, the ledger device naming by scheduling power equipment, the use of fuzzy matching technology and equipment grid companies or asset management 
information system equipment account were correspondence [5].

Take Guangzhou power grid for example, business collaboration and realization of grid technology route scheduling and other business units of the grid is shown in Figure 2:

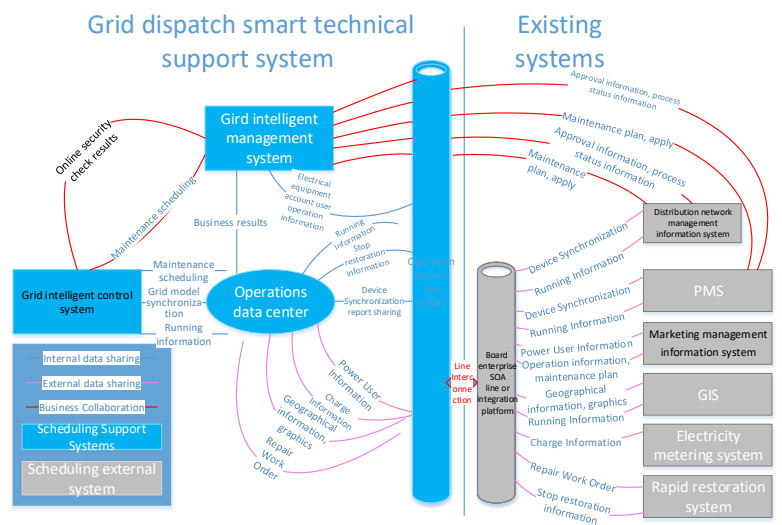

Figure 2. Business Collaboration Logic

It is mainly achieved by running the bus service with existing systems for data exchange and business process reengineering and choreography, supporting business collaboration.

Set electrical equipment as the main line to achieve the integration information and management information, and establish the data center covers main distribution network operation information panorama; combine distributed file system (HDFS) and distributed relational database storage technology to achieve mass data storage grid operation; combine GIS technology and mobile application technology to achieve a hierarchical partition display grid.

On the basis of data integration and distributed storage, according to the analysis of various types of electric power scheduling scenarios, design power grid data analysis and calculation framework that provides power flow analysis, accident inversion, dynamic simulation, analysis and other support equipment status.

In the secondary security protection of power and the national overall framework, enterprise and power grid safety protection requirements, design and build electric power dispatching mobile application platforms. Safety, design and implementation of a unified security access and credit access system, using network licensing, equipment legitimate, legitimate authority and a legitimate user confirmation quadruple security authentication measures.

\section{Conclusions and Future Work}

The author takes Guangzhou Power Grid as a typical large city newly established power grid, analysis the situation and development of power grid in Guangzhou, combined with the rapid development of information technology, smart grid technology and other technologies, proposed grid dispatch technical support system development direction and reasonable plan for its construction work, building a clear technology roadmap and implementation of content and typical problems. In this paper, the technology system planning, technical route and problems typical realization method has been applied to the the construction of power grids in Guangzhou and it has been demonstrated.

\section{References}

1. Liang Shou-yu, Hechao Lin, Xiong Weibin, and so on, China Southern Power Grid scheduling information "Twelfth Five Year Plan" [R], Guangzhou: China Southern Power Grid Co., Ltd., 2011.

2. WANGJi-feng. A Preliminary Investigation on Development of Operation Smart System for China Southern Power Grid[J].Southern Power System Technology, 2012,6(2):1-5.

3. YANGKun, WUXiao-fei, et al. Coping strategy for the situation faced by Guangzhou Twelfth Five-Year power network plan and construction. Technological Development of Enterprise, 29(21):123-124.

4. WU Ren-bo. The Design and Implementation of Intelligent Dispatching Technical Support Platform Base on main Distribution Network Cooperation. Shan Dong: Shan Dong University, 2013.

5. LI Feng,LIU Zheng-chao,et al. Study and Application of Integrated Power System Planning Information System in Guangdong[J]. Proceedings of the CSU-EPSA. 